\title{
Association of progressive outer retinal necrosis and varicella zoster encephalitis in a patient with AIDS
}

\author{
G J van den Horn, C Meenken, D Troost
}

\begin{abstract}
Background-A patient with AIDS who developed the clinical picture of bilateral progressive outer retinal necrosis (PORN) in combination with varicella zoster encephalitis is described. The picture developed more than 2 years after an episode of ophthalmic zoster infection, and following intermittent exposure to oral acyclovir because of recurrent episodes of cutaneous herpes simplex infection.

Methods-Aqueous humour, obtained by paracentesis of the anterior chamber, was analysed using immunofluorescence and polymerase chain reaction (PCR). Postmortem analysis of eye and brain tissue was performed by using conventional techniques and in situ hybridisation.

Results-While conventional techniques all failed to detect a causative agent, analysis of the aqueous humour using PCR, and histological examination of necropsy specimens from eyes and brain using in situ hybridisation were conclusive for the diagnosis varicella zoster virus (VZV) infection.

Conclusion-This case documents the presumed association of PORN and VZV encephalitis in a severely immunocompromised AIDS patient.
\end{abstract}

(Br f Ophthalmol 1996;80:982-985)

Necrotising retinitis due to varicella zoster virus $(\mathrm{VZV})$ in patients with severe immunoincompetence in the acquired immunodeficiency syndrome (AIDS) is regarded as a distinct clinical entity. ${ }^{1-3}$ It is characterised by a multifocal outer retinitis, followed by a fulminant progression of outer retinal necrosis. For this reason, the syndrome is often referred to as progressive outer retinal necrosis syndrome (PORN). Virtually no vitreitis or vasculitis is seen. Funduscopy shows a pale oedemic posterior pole, with a cherry red spot appearance of the fovea. Often, a perivascular sparing, or clearing, may be seen. In the majority of the cases the fellow eye is also involved. Visual outcome is usually extremely poor, irrespective of antiviral treatment. Retinal detachments have been reported as a complication. ${ }^{3}$

The clinical picture of this syndrome is different from the acute retinal necrosis syndrome (ARN) which occurs in immunocompetent individuals. The former syndrome shows less iridocyclitis, vitreitis, and vasculitis, while the initial lesions tend to occur in the posterior pole rather than in the retinal periphery. Both syndromes have in common that they are often found to occur following an episode of cutaneous zoster infection. Recently, Kuppermann and associates ${ }^{3}$ observed an additional new feature that comprises the clinical syndrome: the enhanced risk of encephalitis. In this paper, we present a case of PORN caused by VZV in a patient with AIDS, who also had a histologically proved VZV encephalitis. Using more advanced techniques we were able to confirm the diagnosis, while conventional techniques all failed to do so.

\section{Methods}

Paired serum and aqueous humour samples obtained by paracentesis of the anterior chamber were analysed for antibodies against Toxoplasma gondii, herpes simplex virus (HSV), VZV, and cytomegalovirus (CMV). The respective Goldmann-Witmer coefficients were calculated. ${ }^{45}$ Aqueous humour was also analysed for viral and parasitic DNA using polymerase chain reaction (PCR) ${ }^{67}$

Specimens of aqueous, vitreous, iris, retina, brain tissue, and cerebrospinal fluid obtained post mortem were used for viral and bacterial cultures, and direct preparations. Additionally, PCR techniques were used for detection of viral and parasitic DNA. Brain tissue was also analysed by in situ hybridisation (standard DNA-ISH technique).

\section{CASE REPORT}

A 36-year old man with AIDS (HIV antigen tested positive in 1987) presented in January 1994 with a rapidly progressive painless loss of vision in both eyes. In September 1991 he had an episode of right sided ophthalmic zoster, which was treated with high doses of oral acyclovir and locally applied acyclovir ointment. Viral culture of fluid obtained from a vesicle yielded varicella zoster virus. At that time, the CD4+ lymphocyte count was 20 cells $\times 10^{6} / 1$. In December 1991 he was hospitalised with symptoms of acute right hemiparesis and dysphasia, shortly followed by a left sided paresis. Computed tomography (CT) scans of the brain showed multiple hypodense lesions, which increased in time. Arteriography of the carotid arteries showed alterations of the vascular walls, which were interpreted as probably due to cerebral vasculitis. The symptoms appeared to be transitory, and the patient refused further investigations before a thorough diagnosis was made. In December 1992 the Netherlands. 28 June 1996 


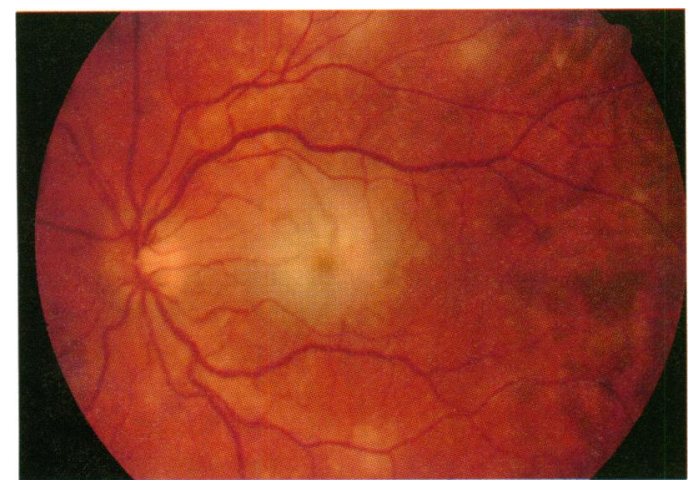

Figure 1 Progressive outer retinal necrosis (left eye). Early in the disease, the posterior pole shows a pale oedemic area with a cherry red spot appearance of the macula. In the mid periphery, multiple small yellowish infiltrates can be seen, that become confluent in the later stages.

and December 1993 he had short episodes of similar transient neurological symptoms; the lesions found on CT scans and magnetic resonance images (MRI) of the brain were interpreted as infarctions caused by vasculitis.

From January 1992 until July 1993 he experienced intermittent episodes of ophthalmic complaints, due to varicella zoster keratouveitis: the right eye showed corneal stromal infiltrations and cells in the anterior segment, followed by atrophia of the iris. Visual acuity of this eye diminished to $20 / 30$. Treatment consisted of topical atropine, steroids, and TFT or acyclovir. No retinal infiltrations were seen. The left eye remained unaffected, with a visual acuity of $20 / 20$.

From July 1993 until January 1994 the patient was free of ophthalmic complaints. During that period, he intermittently used low dosages of oral acyclovir because of eruptions of herpes simplex virus type II in the anal region.

The symptoms of progressive bilateral visual loss were associated with behavioural disturbances. The patient was euphoric and uncontrolled, and exhibited a loss of decorum.

Examination of the eyes yielded a best corrected visual acuity of $20 / 200$ in the right eye and counting fingers $(1 / 60)$ in the left. Slitlamp examination showed no signs of corneal

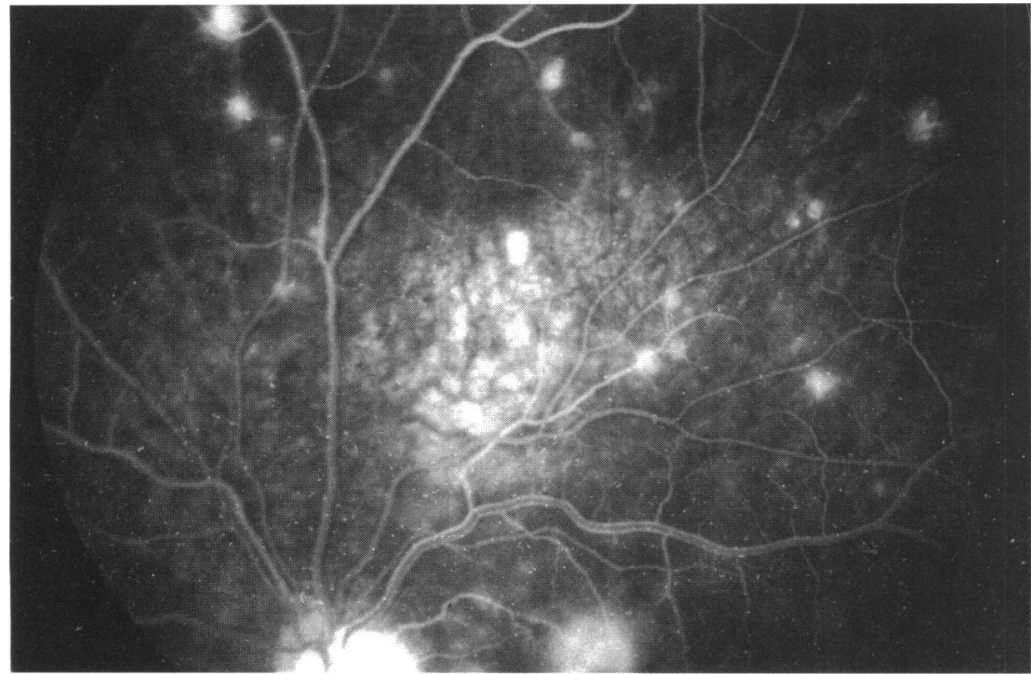

Figure 2 Fluorescein angiography (left eye) shows infiltrations in the deep retinal layer. The vasculature is normal; no signs of vasculitis are present.

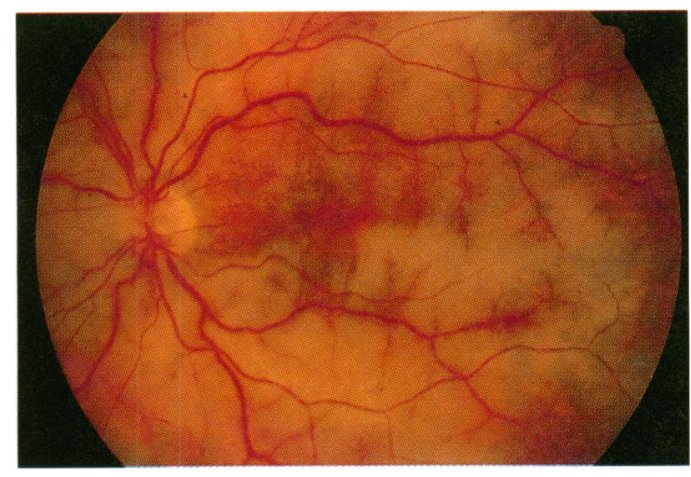

Figure 3 Progressive outer retinal necrosis (left eye), 10 days after the stage of Figure 1. Perivascular clearing: while the retinitis progresses, oedema is cleared in the perivascular areas.

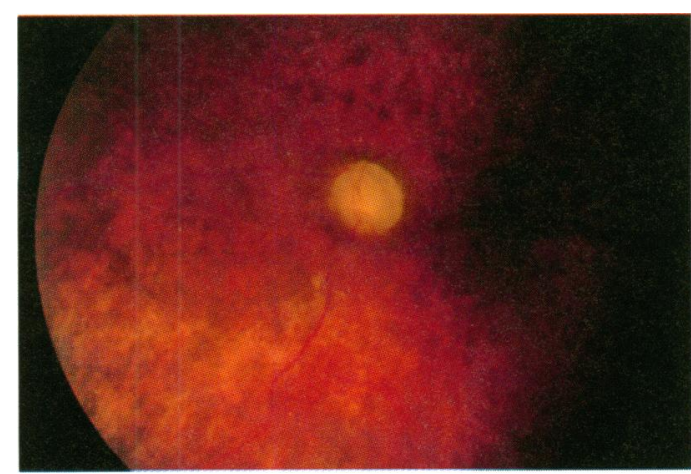

Figure 4 Progressive outer retinal necrosis (left eye). Ultimately, the retina becomes burned out: note atrophic areas and destruction of the vasculature. Optic disc pallor can be seen.

lesions, nor inflammation of the anterior segments or the vitreous. Funduscopy, however, showed pale oedemic posterior poles, with a cherry red spot appearance of the maculas. In the periphery, several small deep yellowish infiltrations could be seen (Fig 1). These spots showed hyperfluorescence in the later phases on the fluorescein angiograms. There were no signs of vasculitis (Fig 2). Retinal necrosis progressed, showing the typical aspect of perivascular clearing (Fig 3).

The visual acuity of both eyes deteriorated rapidly, and the patient became totally blind within 2 weeks, despite treatment with acyclovir. The chorioretinitis became burned out, and atrophic retinal scars were formed (Fig 4). Retinal detachment did not occur. Seven weeks later, the patient died at home. We were allowed to use the eyes and the brain for postmortem analysis.

\section{Results of diagnostic procedures and necropsy}

Antibody analysis in aqueous from the right eye yielded no Goldmann-Witmer coefficients above 1 for Toxoplasma gondii, HSV, CMV, or VZV.

Viral and bacterial cultures from eye specimens at necropsy all remained negative. In the direct preparations no parasites or bacteria could be detected. However, VZV DNA could be detected by PCR in all specimens, whereas $\mathrm{CMV}, \mathrm{HSV}$, and $T$ gondii remained negative.

At necropsy the brain showed multifocal cystic lesions with central necrosis (Fig 5). These foci ranged from 3 to $25 \mathrm{~mm}$ in 


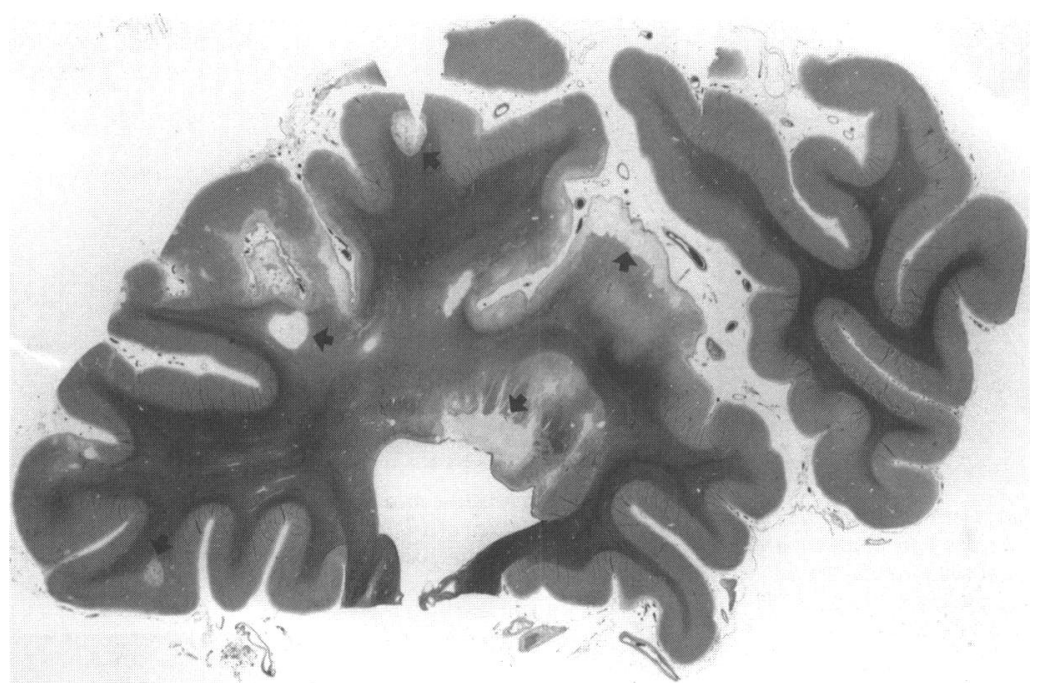

Figure 5 Coronal section of the right parietal lobe. Several large and small necrotic foci (arrows) in the white matter. Some lesions mimic laminar necrosis. The central white matter is mildly demyelinated. tis in their patients was related to the coinciding ophthalmic zoster infection rather than to the retinitis.

The visual prognosis of PORN is extremely poor. Although recently a possible beneficial effect of oral sorivudine has been reported, ${ }^{13}$ treatment with antivirals such as ganciclovir, foscarnet, or acyclovir has been shown to be quite ineffective to date. ${ }^{14}$ There is some evidence that combination antiviral therapy instead of monotherapy may delay progression of the retinitis. ${ }^{15}$

Immunocompromised patients with a history of ophthalmic zoster have an enhanced risk of developing VZV retinitis, ${ }^{16}$ even after a long period of time. The patient reported here shows an interval of 27 months between the ophthalmic zoster infection and the outbreak of PORN despite an extremely low CD4 count. This raises the question whether such patients should be on a prophylactic regimen and receiving antivirals. However, AIDS patients usually have been exposed to several antiviral drugs during their illness. It should be kept in mind that this may eventually lead to drug resistant strains of the virus. VZV encephalitis simultaneously with PORN may develop as part of a disseminated VZV disease indicating total depletion of the immune system. In that case, it can be an ominous sign of a disease for which effective treatment is as yet an illusion.

We thank the coworkers of the Department of Ophthalmoimmunology of the Netherlands Ophthalmic Research Institute for Goldmann-Witmer and PCR analyses.

The patient reported here shows the clinical syndrome of PORN in extremely immunocompromised individuals. In addition, the association of this syndrome with encephalitis is illustrated in our patient. The presumed aetiological role of VZV was confirmed by clinical and postmortem observations. We were able to detect the virus by means of PCR and in situ hybridisation techniques.

The long interval between first and final manifestations of VZV infection in this patient allowed us to distinguish between the subsequent neurological complications of ophthalmic zoster infection and their respective associations, in relation to a deteriorating immune system: cerebral infarctions, a well known complication of ophthalmic zoster, are attributed to cerebral vasculitis. ${ }^{38-10}$ This condition could be confirmed radiographically at the time of the transient hemiplegias; the reported form of necrotising retinitis with encephalitis is related to a deeper immune depletion. VZV encephalitis was confirmed, but no signs of cerebral vasculitis could be demonstrated in the necropsy specimens. Vascular abnormalities in patients with terminal AIDS and VZV encephalopathy are rare, ${ }^{11}$ and the absence of histological evidence of cerebral vasculitis in this stage of the disease is not surprising. It illustrates the inability to mount effective inflammatory responses and reflects the deep immunoincompetence of patients suffering from this particular form of retinitis.

Rousseau et al ${ }^{12}$ reported three patients with VZV retinitis in association with cerebral vasculitis. We believe that the cerebral vasculi-
1 Forster DJ, Dugel PU, Frangieh GT, Liggett PE, Rao NA. Rapidly progressive outer retinal necrosis in the acquired 110:341-9.

2 Margolis TP, Lowder CY, Holland GN, Spaide RS, Logan $\mathrm{AG}$, Weissman SS, et al. Varicella-zoster virus retinitis in patients with the acquired immunodeficiency syndrome. Am f Ophthalmol 1991;112:119-31.

3 Kuppermann BD, Quiceno J, Wiley C, Hesselink J, Hamilton $\mathrm{R}$, Keefe $\mathrm{K}$, et al. Clinical and histopathologic study of immunodeficiency syndrome. Am $₹$ Ophthalmol 1994;118: 589-600.

4 Luyendijk L, Van den Horn GJ, Visser OHE, SuttorpSchulten MSA, Kijlstra A. Detection of locally produced antibodies to herpes viruses in the aqueous humour of patients with acquired immunodeficiency syndrome (AIDS) or acute retinal necrosis syndrome (ARN). Curr

5 De Boer JH, Luyendijk L, Rothova A, Baarsma GS, De Jong PVTM, Bollemeijer JG, et al. Detection of intraocular antibody production to herpesviruses in acute retinal necrosis syndrome. Am ₹ Ophthalmol 1994;117:201-10.

6 Fox GF, Crouse CA, Chuang EL, Pflugfelder SC, Clearly TJ, Nelson SJ, et al. Detection of herpes virus DNA in vitTJ, Nelson SJ, et al. Detection of herpes virus DNA in vitreous and aqueous specimens by the polym
reaction. Arch Ophthalmol 1991;109:266-71.

7 Aouizerate F, Cazenave J, Poirier L, Verin P, Cheyrou A, Begueret J, et al. Detection of Toxoplasma gondii in aqueous humour by the polymerase chain reaction. $\mathrm{Br} f$ Ophthalmol 1993;77:107-9.

8 Frank Y, Lim W, Kahn E, Farmer P, Gorey M, Pahwa S. Multiple ischemic infarcts in a child with AIDS, varicella zoster infection and cerebral vasculitis. Pediatr Neurol 1989; 5:64-7.

9 Doyle PW, Gibson G, Dolman CL. Herpes zoster ophthalmicus with contralateral hemiplegia. Identification of cause. Ann Neurol 1983;14:84-5.

10 Gray F, Bélec L, Lescs MC, Chrétien F, Ciardi A, Hassine $\mathrm{D}$, et al. Varicella zoster virus infection of the central nerv$\mathrm{D}$, et al. Varicella zoster virus infection of the central nervous system in the acquired
Brain 1994;117:987-99.

11 Gray F, Mohr M, Rozenberg F, Bélec L, Lescs MC, Dournon E, et al. Varicella zoster virus encephalitis in acquired immunodeficiency syndrome. Report of four cases. Neuropathol Appl Neurobiol 1992;18:502-14.

12 Rousseau F, Peronne C, Raguin G, Thouvenot D, Vidal A, Leport $\mathrm{C}$, et al. Necrotizing retinitis and cerebral vasculitis due to varicella zoster virus in patients infected with the immunodeficiency syndrome. Am $\mathcal{f}$ Ophthalmol 1990; Eye Res 1990;9(suppl):7-11. 
human immunodeficiency virus. Clin Infect Dis 1993;17: 943-4.

13 Pinnolis MK, Foxworthy D, Kemp B. Treatment of progressive outer retinal necrosis with Sorivudine. $A m$ Ophthalmol 1995;119:516.

14 Batisse D, Eliaszewicz M, Zazoun L, Baudrimont $M$ Pialoux G, Dupont B. Acute retinal necrosis in the course of AIDS: study of 26 cases. AIDS 1996;10:55-60.
15 Galindez OA, Sabates NR, Whitacre MM, Sabates FN Rapidly progressive outer retinal necrosis caused by varicella zoster virus in a patient infected with human immunodeficiency virus. Clin Infect Dis 1996;22:149-51.

16 Sellitti TP, Huang AJW, Schiffman J, Davis JL. Association of herpes zoster ophthalmicus with acquired immunodeficiency syndrome and acute retinal necrosis. Am $\mathcal{f}$ Ophthalciency syndrome and acure. 